\title{
Combined Force-Frequency Sampling for Simulation of Systems Having Rugged Free Energy Landscapes
}

Emre Sevgen, ${ }^{\dagger}$ Ashley Guo, ${ }^{\dagger}$ Hythem Sidky, $^{\dagger}$ Jonathan K. Whitmer, ${ }^{\ddagger}$ and Juan J. de Pablo*,†,

$\dagger$ Pritzker School of Molecular Engineering, University of Chicago, Chicago, Illinois 60637, USA

$\ddagger$ Department of Chemical and Biomolecular Engineering, University of Notre Dame, Notre Dame, IN 46556

ФMaterials Science Division, Argonne National Laboratory, Argonne, IL 60439

E-mail: depablo@uchicago.edu

1 Supplemental Information

1.1 Two-Dimensional Plots of Polymer Diffusion Through a Pore

(A)

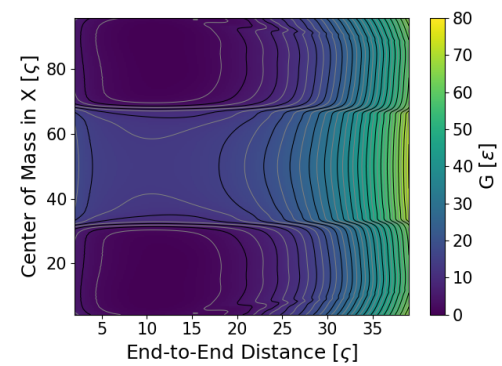

(B)

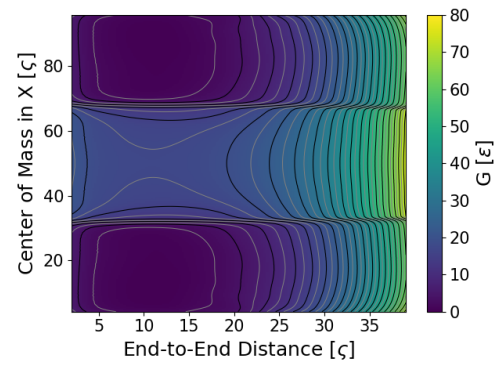

(C)

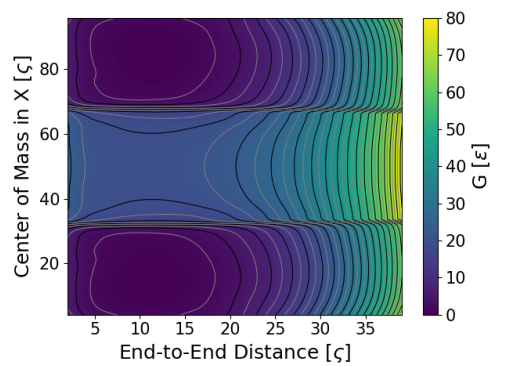

Figure 1: Free energy surfaces for a 50-bead Kremer-Grest polymer diffusing through a pore of A) 10x10, B) $8 \times 8$ and C) $6 \times 6$ at $5.0 * 10^{6}$ LJ timesteps, with the two collective variables defined as the center of mass of the polymer in the pore dimension and end-to-end distance of the polymer. 